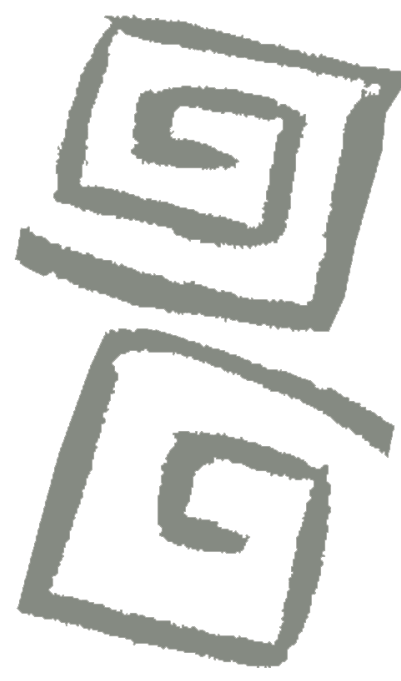

\title{
Cartografiar el tejido de redes de atención para personas con discapacidad, desde la perspectiva de usuarias-ciudadanas-guías
}

\author{
Mapping the intricacies of care networks for \\ persons with disabilities, from the perspectives \\ of users-citizens-guides
}

${ }^{1}$ Autora de correspondencia. Doctora en Salud Pública. Profesora Asociada Departamento de Promoção da Saúde, Centro de Ciências Médicas, Universidade Federal da Paraíba, João Pessoa, Paraíba, Brasil. $\triangle$ (iD)

${ }^{2}$ Doctora en Educación. Profesora Adjunta, Departamento de Promoção da Saúde, Centro de Ciências Médicas, Universidade Federal da Paraíba, João Pessoa, Paraíba, Brasil. $\triangle$ iD

${ }^{3}$ Médico, Doctor en Clínica Médica, Profesor Adjunto, Departamento de Promoção da Saúde, Centro de Ciências Médicas, Universidade Federal da Paraíba, João Pessoa, Paraíba, Brasil. $\triangle$ (iD)

${ }^{4}$ Psicóloga clínica. Magíster en Psicología, Universidade de Pernambuco, João Pessoa Paraíba, Brasil. $\triangle$ iD

${ }^{5}$ Maestrando en Programa de Pós-graduação em Saúde Coletiva, Universidade Federal da Paraíba; João Pessoa, Paraíba, Brasil. $\triangle$ id

${ }^{6}$ Estudiante de Medicina, Universidade Federal da Paraíba, João Pessoa, Paraíba, Brasil. $\square$ (iD)

${ }^{7}$ Estudiante de Medicina, Universidade Federal da Paraíba, João Pessoa, Paraíba, Brasil. $\square$ (iD)
Juliana Sampaio', Daniella de Souza Barbosa², Luciano Bezerra Gomes ${ }^{3}$, Daniele Alves Peixoto ${ }^{4}$, Tarcísio Almeida Menezes ${ }^{5}$, Hariel Hegel Lins Zózimo $^{6}$, Maria Fernanda de Britto Lyra

RESUMEN En este artículo analizamos la producción de redes de atención para personas con discapacidad, en el estado de Paraíba, Brasil. Se trata de un estudio cualitativo, con enfoque cartográfico, en el que participaron cuatro usuarias-ciudadanas-guías. Se realizaron reuniones virtuales semanales a lo largo del segundo semestre de 2020, en las que pudimos recolectar, sistematizar y producir consistencia teórica, a partir de cuatro analizadores: 1) culpabilidad y (no) esperanza de vida de la persona con discapacidad; 2) el lugar de la discapacidad en la dinámica familiar; 3) la producción de núcleos de dependencia relacional específicos; y 4) el público vs. ofertas privadas de atención para las personas con discapacidad. A partir de ellos, fue posible producir visibilidades y enunciabilidades sobre las formas en que estas usuarias-ciudadanas son parte del complejo proceso de construcción de cuidados para las personas con discapacidad y sus familias. PALABRAS CLAVES Servicios de Salud para Personas con Discapacidad; Necesidades y Demanda de Servicios de Salud; Modelos de Atención de Salud; Relaciones Familiares; Investigación Cualitativa; Brasil.

ABSTRACT This article presents an analysis of the production of care networks for persons with disabilities in the state of Paraíba, Brazil. We employed a qualitative methodology with a cartographic approach, involving the participation of four users-citizens-guides. Weekly virtual meetings were held throughout the second half of 2020, in which we were able to collect, systematize, and theorize on four issues: 1) guilt and (non) life expectancy on the part of persons with disabilities; 2) the place of disability in family dynamics; 3 ) the production of specific core relational dependency networks; and 4) the public vs. private care offerings for persons with disabilities. Based on these findings, we were able to lend visibility to and explicitly affirm the ways in which these user-citizens participate in the complex process of care building for persons with disabilities and their families.

KEY WORDS Health Services for Persons with Disabilities; Health Services Needs and Demand; Healthcare Models; Family Relations; Qualitative Research; Brazil. 


\section{INTRODUCCIÓN}

La organización de redes de asistencia de salud ha sido una estrategia común para muchos sistemas de salud universales durante años $^{(1)}$. Sin embargo, existe una excesiva centralidad en su formulación, implementación y evaluación desde la perspectiva de los trabajadores y directivos. Es fundamental reflexionar sobre los cambios que podrían producirse en el campo al comprender cómo se producen los cuidados en redes, haciendo más visible el punto de vista de los usuarios. Recurrimos a la noción de "punto de vista" para poner en cuestión no una diferencia de opiniones sobre un mismo objeto, sino un desplazamiento de perspectivas, noción que permite entender la disputa en torno a la construcción de mundos distintos, a partir de la negociación que se establece entre seres en relación ${ }^{(2,3)}$. De ahí la necesidad de considerar a los usuarios como ciudadanos, cuyas trayectorias pueden guiarnos en la comprensión de los complejos procesos relacionados con la producción de cuidados $^{(4)}$. Esto los sitúa no solo en el lugar de evaluadores, cuya satisfacción debemos medir y cuyas opiniones y denuncias debemos recoger a través de mecanismos como auditorías, sino que los reconoce como productores de cuidado en redes, en las cuales los servicios formales de salud son agenciados y agenciadores, pero no limitan sus trayectorias ${ }^{(5,6)}$

En este sentido, consideramos pertinente reflexionar sobre la producción de cuidados en red desde otra perspectiva, en la que a la condición de usuarios-ciudadanos, le sumamos el lugar de las personas con discapacidad. La singularidad con la que estas personas construyen sus proyectos de futuro para sí mismas y necesitan disputarlos, muchas veces invisibilizados, aporta aspectos importantes para repensar no solo cómo se organiza la atención de la salud de las personas con discapacidad, sino cómo se realiza el cuidado en los servicios en general ${ }^{(7)}$. Por ello, en el presente artículo buscamos analizar las producciones de las redes de cuidado para personas con discapacidad, a partir de las usuarias-madres-ciudadanas que tomamos como guías en un recorrido cartográfico.

Se trata de un recorte de la investigación "Análise da implementação da rede de cuidados à saúde das pessoas com deficiência - os usuários, trabalhadores e gestores como guias", financiada por el Conselho Nacional de Desenvolvimento Científico e Tecnológico $(\mathrm{CNPq})$, llevada a cabo por la red de Observatórios de Políticas Públicas, Educação e Cuidado em Saúde. En el estado de Paraíba, la red de personas con discapacidad fue cartografiada por miembros del Grupo de Pesquisa Política, Educação e Cuidado em Saúde, del Departamento de Promoção da Saúde, del Centro de Ciências Médicas, de la Universidade Federal de Paraíba.

El artículo contiene tres partes. El primer apartado "El camino se conoce andando", presenta, en términos generales, el itinerario cartográfico de la investigación en el estado de Paraíba, desde sus inicios hasta la actualidad. El segundo apartado, "Rastreando encuentros con usuarias-ciudadanas-guías", narra de manera sucinta las historias de cuatro mujeres de la red de personas con discapacidad. Finalmente, en el apartado "Construyendo algunos analizadores de la producción de redes de cuidados para las personas con discapacidad", discutimos los analizadores que se conectan entre los relatos de las cuatro usuarias-ciudadanas-guías y que producen significados en nuestro recorrido cartográfico.

\section{EL CAMINO SE CONOCE ANDANDO}

En 2019, primer año de la investigación, las y los integrantes del GPECS realizaron diferentes ingresos al campo, que incluyeron acordar los términos de la investigación con el Consejo de Gestión de la red de personas con discapacidad del estado de Paraíba y un mapeo de los servicios de la red en João Pessoa, capital del estado. Esto permitió identificar el Centro Especializado en Rehabilitación tipo IV (CER IV) de referencia en Paraíba, en servicios para personas con discapacidades 
intelectuales, visuales, auditivas, físicas, múltiples, trastornos del espectro autista, personas con altas capacidades/superdotadas y personas diagnosticadas con esclerosis múltiple.

Inicialmente, compartimos las vivencias de la vida cotidiana del servicio, se elaboraron registros cartográficos en diarios de campo, y se organizaron reuniones quincenales con sus responsables y trabajadores. En estos encuentros se generó un espacio de educación permanente en salud, que involucró a participantes de diversas coordinaciones y núcleos del servicio. Las diez reuniones de educación permanente en salud tuvieron lugar en el segundo semestre de 2019, con una media de diez participantes de los 18 inscritos, y se caracterizaron por ser espacios horizontales de intercambio de experiencias y de construcción de posibilidades de acción entre las y los trabajadores.

En 2020, con la pandemia de COVID-19, adaptamos nuestros recorridos por la red de personas con discapacidad al contexto de la distancia física. Los recursos tecnológicos de comunicación e información fueron aliados para continuar las reuniones, ahora virtuales y mensuales, con trabajadores y directivos de la institución. También ampliamos nuestra cartografía a partir de las usuarias-ciudadanas-guías, a través de reuniones mediadas por WhatsApp y plataformas como Google Meet. Todos los encuentros e intercambios se registraron en diarios cartográficos, y obedecieron a los requisitos éticos en la investigación con seres humanos: se obtuvo el consentimiento de los participantes, se garantizó su confidencialidad y la investigación fue aprobada por el Comité de Ética en Investigación, bajo el CAAE: 17725919.2.1001.5699.

\section{Rastreando encuentros con usuarias- ciudadanas-guías}

La primera de las usuarias-ciudadanas-guías de nuestra cartografía es Rita, madre de un niño con trastorno del espectro autista que accede al CER IV para obtener certificados clínicos en busca de efectivizar el derecho de su hijo a la asistencia social, pero que no utiliza los servicios de rehabilitación intelectual que allí se ofrecen.

Nuestra conexión con Rita surgió del contacto previo de uno de los investigadores. Al comentarle sobre la red de personas con discapacidad, el investigador escuchó a Rita contar qué es llevar una vida siendo madre de un niño con trastorno del espectro autista. Así, se evidenciaron los puntos en común entre su historia de vida, el CER IV y la red local de personas con discapacidad. Luego, oficializamos el contacto con Rita y se convirtió en una de las madres-ciudadanas-guía de nuestra cartografía.

Rita es una mujer a la que le gusta el arte y cuidar de los demás. Se graduó en educación artística y, posteriormente, obtuvo una segunda formación en el área de la salud. Con muchos deseos y aspiraciones, reconoce en la educación una forma de ofrecer a su familia nuevas posibilidades, lo que la hizo ingresar a un programa de maestría y nutrir el sueño de llegar a ser profesora universitaria.

Su marido y sus dos hijos viven con ella. El mayor es un niño de cinco años diagnosticado con trastorno del espectro autista, al que Ilamaremos, en este texto, Joaquim. Al enterarse del diagnóstico de su hijo, nuestra madre-ciudadana-guía buscó acercarse a otras madres que estuvieran pasando por la misma experiencia, y terminó participando en varios grupos de WhatsApp de madres de niños con trastorno del espectro autista.

Para definir el diagnóstico de Joaquim, Rita accedió a las redes de salud privada y pública, y tuvo éxito en ambas. Realiza terapias psicológicas y psicopedagógicas, a las que ha accedido a través del plan de salud privado. Actualmente, está de vuelta en el CER IV para evaluar la posibilidad de que Joaquim tenga también altas capacidades/superdotación, es decir, doble excepcionalidad. Esta condición puede definirse como la presencia de un alto rendimiento, talento, capacidad o potencial, que se produce junto con un trastorno psicológico, conductual, neurológico, psiquiátrico, educativo, sensorial o físico ${ }^{(8)}$.

La segunda usuaria-ciudadana-guía -a la que llamaremos Margarida- es una mujer de 48 años, casada, cuidadora de niños y ancianos, 
actualmente desempleada. Margarida tiene un hijo adulto, vive en la periferia de João Pessoa y es usuaria del CER IV por tener baja visión, siendo atendida en el sector de rehabilitación visual (CODAVI). Llegó a nosotros a través de las narrativas de uno de nuestros investigadores que acompaña el grupo de WhatsApp de usuarios del CODAVI, y que siempre se refería a ella como una de las personas más activas del grupo.

Nos pusimos en contacto con ella mediante mensajes de texto, pudimos intercambiar información sobre la investigación y nos reunimos virtualmente para conocer su historia. Tiene diabetes mellitus tipo 2, y descubrió tal condición tras lesionarse el pie con un clavo; al no ver ni sentir el traumatismo, presentó complicaciones y tuvo que permanecer unos días ingresada en el hospital para el tratamiento de la herida y el control de la alta tasa de glucemia que presentaba. Después de cuatro meses de esta hospitalización, empezó a sentir la visión borrosa y tuvo que ser operada para evitar la ceguera. Esta cirugía se realizó en la red privada, gracias a que uno de sus hermanos le prestó la cantidad de $\mathrm{R} \$ 10.000$ reales. Pero el procedimiento no funcionó y, tras volver al oftalmólogo, este la remitió al Sistema Único de Salud (SUS), ya que no tenía más dinero para someterse a otra operación. Margarida, entonces, pensó: "si en la red privada no funcionó, en el SUS, que es gratuito, ino funcionará en absoluto!". Pero fue gracias a esta segunda operación, realizada en el sistema de salud pública, que pudo recuperar parte de la visión de un ojo.

Margarida empezó a acceder al CER IV cuando se enteró de que tenía derecho al abono gratuito del transporte público de la ciudad, porque a menudo no tenía dinero para el boleto, lo que le dificultaba incluso ir al servicio de salud. En el CODAVI, ya pasó por los servicios de rehabilitación (formación en actividades de la vida diaria) y habilitación (formación para el uso de un bastón). Continúa vinculada al servicio para recibir atención psicológica. Accede a la red básica, en una unidad cercana a su casa, para medir la glucosa y realiza actividades físicas intermitentes en el programa Gimnasio de Salud, cerca de donde vive. La derivaron a un control nutricional en el Hospital Universitario y se le sugirió una cirugía bariátrica, pero con la pandemia ya no volvió a las consultas.

Siente mucha culpa por no haber cuidado de su salud antes y consumir una dieta más sana, porque cree que, de esta forma, habría evitado perder parte de su visión. Todavía no consigue controlar sus niveles de azúcar en sangre y sigue escuchando por parte de los médicos que debe cuidarse para no perder la vista por completo. No le gusta hacer actividad física y su alimentación está limitada por el bajo poder adquisitivo del salario mínimo de su marido, que trabaja como barrendero. Margarida es una mujer vanidosa y le entristece no trabajar para tener su propio dinero, para comprar ropa interior, por ejemplo. Relata que su sueño es participar de un programa de entretenimiento de transformación de belleza que se emite los domingos por la tarde en una cadena de televisión nacional, pidiendo ayuda para inscribirse en él.

A continuación, presentamos a una pareja, nombrada por nosotros como Maria y José, ambos profesionales de la salud, con militancia en el SUS desde el movimiento estudiantil. Después de participar en la gestión sanitaria municipal en su ciudad, se trasladaron a Brasilia para ocupar espacios de gestión a nivel federal de importantes políticas en las áreas en las que trabajaban. Se encontraban en un momento de intensa apuesta por la construcción de políticas públicas, que se vería coronada con el embarazo de su primer hijo, al que llamaremos João.

En los primeros meses, a pesar del cansancio y los desencuentros de quienes se encontraban en su primera pater-maternidad, la vida era intensa. Hasta que algunos de los indicadores de crecimiento y desarrollo del niño empezaron a ser diferentes a lo que se esperaba para su edad, aspecto señalado inicialmente por una de las abuelas. La identificación de que João tenía trastorno del espectro autista llevó a una efectiva revolución en la vida de toda la familia que se movilizó, superponiendo y reconstruyendo conocimientos, afectos y proyectos. 
Se desencadenó un intenso proceso para superar el desconocimiento de los padres y así hacer frente a la falta de preparación de los profesionales involucrados en el cuidado de João; el regreso a la tierra natal, debido a la falta de condiciones en los servicios públicos para atender al niño y a las redes de apoyo social en Brasilia, la gran dificultad para identificar servicios capaces de proporcionar una atención adecuada, y las barreras para acceder a la escuela regular debido a la falta de preparación pedagógica y a los prejuicios de las instituciones y de los padres de otros niños.

La familia se vio marcada por las intersecciones de género y clase que las llevó a transitar otras facetas en el contexto de las familias de personas con discapacidad. Se depararon con el uso medicinal del extracto de Cannabis ssp. como una posibilidad y una barrera para tratar las convulsiones de João $y$, en todo esto, comenzaron a valorar cada pequeña victoria.

Finalmente, llegamos a Glória y a su tercer hijo Bentinho -también nombres ficticiospor indicación del coordinador del Servicio Especializado en Rehabilitación Intelectual de la Coordinación de Atención a Personas con Trastorno del Espectro Autista (CODEI/SERI) del CER IV. Bentinho, un preadolescente de 11 años, es usuario del servicio desde 2011.

Durante nuestros encuentros por videoconferencia, Glória recordó su vida como mujer, madre, esposa, trabajadora y activista social (revelando su multiplicidad), cómo su vida y la de su familia quedó marcada por la Ilegada de su tercer hijo, Bentinho, en 2009, y su paso por la red local de personas con discapacidad.

Ella afirma que Bentinho se desarrolló "con normalidad" hasta los dos años, llegando incluso a balbucear sus primeras palabras. Sin embargo, todavía en el segundo año de su vida, Bentinho sufrió un accidente doméstico: al tirar de una sartén que estaba en la cocina con agua hirviendo, sufrió graves quemaduras en casi un $40 \%$ de su cuerpo. Al tener que pasar un mes en una unidad de terapia para quemados, Bentinho se volvió "terco", berrenchín, agresivo y distante, especialmente en espacios abiertos y lugares concurridos, además de retroceder en la adquisición del habla.

Ante este cambio de comportamiento, y comparando a Bentinho con sus otros dos hijos cuando tenían la misma edad, Glória percibió "algo diferente" en él, vinculado no necesariamente al episodio de la quemadura. A partir de ese momento, Bentinho pasó por el triaje y la evaluación multiprofesional (con psicólogo, psiquiatra y fonoaudiólogo) en el CODEI/SERI del CER IV. Con el diagnóstico de trastorno del espectro autista, comenzó a realizar actividades de fonoaudiología, psicoterapia y musicoterapia en este servicio.

Sin embargo, Glória estaba disconforme con el servicio del CER IV porque no satisfacía todas las necesidades de Bentinho, especialmente en lo que respecta a la adquisición del habla, ya que cada sesión duraba solo 30 minutos y ella tenía poco acceso a los rehabilitadores y ningún conocimiento de cuál era el plan terapéutico. Glória también sintió la necesidad de que sus necesidades como madre de una persona autista sean acogidas, pero no se le ofreció. Para colmo, desvincularon a Bentinho de musicoterapia, la actividad que más le gustaba, con la excusa de que el servicio "tenía que abrir una vacante para nuevos usuarios".

Al consultar la red privada de salud, Glória se encuentra con la barrera económica de acceso: los costos mensuales serían de unos $\mathrm{R} \$ 2.000$ reales. En 2011, fundó entonces una asociación de madres de personas con trastorno del espectro autista, que desarrolla actividades lúdicas y de rehabilitación para niños y donaciones de canastas de alimentos para más de 35 familias inscritas, formadas en su mayoría por "madres solteras". Glória y su familia -su marido y sus dos hijos mayores, todos ellos estudiantes de Psicologíason muy activos en la asociación, y solicitan a entidades públicas y privadas donaciones de alimentos, juguetes y entradas para actividades culturales, por ejemplo. A partir de estos diversos caminos de la red local de personas con discapacidad, guiados por las manos de estas madres-ciudadanas-guías y sus hijos/ familiares, es posible destacar algunos analizadores de la producción de redes de atención a las personas con discapacidad de Paraíba. 


\section{ANALIZADORES DE LA PRODUCCIÓN DE REDES DE CUIDADOS}

A partir de los encuentros con las usuariasciudadanas-guías, esbozamos algunos analizadores sobre la producción de redes de atención para personas con discapacidad, que conforman nuestra experiencia cartográfica. Entre ellos, destacamos cuatro que queremos compartir: 1) la culpa y la (no) expectativa del poder de producción de vida de las personas con discapacidad; 2) el lugar de la discapacidad en la dinámica familiar; 3 ) la producción de núcleos específicos de dependencia relacional; y 4) las imbricaciones público vs. privado en las ofertas de cuidados para las personas con discapacidad.

\section{La culpa y la (no) expectativa del poder de producción de vida de las personas con discapacidad}

El encuentro con las particularidades del desarrollo del niño y, posteriormente, con el diagnóstico de discapacidad, Ilevan a la familia a cuestionarse por qué están viviendo esa situación. Estas cuestiones conducen casi naturalmente a la búsqueda de una causa, de una justificación que, en la mayoría de las veces, sitúa a la madre en el lugar de responsable/culpable de la deficiencia del hijo que ha generado y dado a luz. Por supuesto, esa culpa tiene fuertes marcas del patriarcado que asigna a las mujeres la responsabilidad de la procreación (y el control reproductivo), y del cuidado de la prole ${ }^{(9)}$. No es raro escuchar relatos que refuerzan la idea de que la mujer fue incapaz de tener hijos (y no la pareja, incluso con las primeras pruebas de infertilidad siendo realizadas en ella), o era incapaz de tener hijos de un determinado sexo. En esta producción discursiva, se culpa fácilmente a la mujer de generar un hijo con discapacidad $^{(10)}$.

Glória no quería cargar con la culpa de la discapacidad de Bentinho sin que se confirmara el diagnóstico. Por lo tanto, buscó el CER IV. Su angustia duró casi un año, el tiempo que tardó en obtener el informe del trastorno del espectro autista de Bentinho. El día que fue a recibir el informe, Glória Ilamó a toda su familia. No podía escuchar la noticia sola (aunque estaba segura del diagnóstico), pues saber que la enfermedad de Bentinho "no tenía cura" dolía demasiado. Para Glória, la culpa materna (apoyada en las construcciones sociales de la maternidad) es aún más incisiva ya que, para ella, Bentinho "se volvió autista" por su vanidad al hacerse una abdominoplastia, aunque no haya evidencia de correlación entre los dos elementos en cuestión.

La culpa materna puede verse reforzada por el propio personal médico y por las teorías elaboradas en el campo de la salud (especialmente en el campo psi) sobre el trastorno del espectro autista. En la experiencia de Maria (una de las madres-guía de esta cartografía), su duelo se agravó, culminando en un cuadro depresivo, cuando los profesionales de la salud que la atendieron recurrieron a la teoría de la madre-heladera, que atribuye el trastorno del espectro autista a disfuncionalidades en la relación afectiva madre-bebé. Basada en teorías de la década de 1940, dicha perspectiva ignora la evolución del conocimiento sobre esta condición, que actualmente identifica en el origen del trastorno del espectro autista factores multicausales: genéticos, biológicos, relacionales, ambientales, culturales, entre otros ${ }^{(11)}$.

Cuando no se atribuye a la madre (en casos de discapacidad congénita o identificada en la primera infancia), o no se reconoce como resultado de un accidente/fatalidad, la culpa de la discapacidad se atribuye entonces al individuo que la porta, como consecuencia de algún comportamiento inadecuado. Este fue el caso de nuestra usuaria-guía-ciudadana Margarida, que tiene una discapacidad visual derivada de una diabetes no controlada. En varios momentos, ella refuerza la idea de sentirse culpable por lo que vive, como si sus dificultades de accesibilidad y de producción de redes de vida fueran exclusivamente resultado de su mal hábito alimentario. Así, no tiene en cuenta las barreras físicas, sociales, 
culturales y políticas que inciden en sus dificultades para caminar por la vida, e incluso para haber mantenido ciertos hábitos alimentarios. Este aspecto también está relacionado con los procesos sociales de expansión de las formas de existencia capitalistas, que pasan por la biomedicalización de la vida ${ }^{(12)}$.

En el ámbito de la responsabilidad de la atención, también entran en juego construcciones sociales que no reconocen la potencia de la producción de vida de las personas con discapacidad. El diagnóstico de un niño con discapacidad está atravesado por el imaginario social de que este ser no será capaz de producir suficiente autonomía para conducir su propia vida, necesitando siempre la tutela de otros ${ }^{(13)}$.

Así, la primera y más destacada preocupación y angustia de Glória provino de los relatos estereotipados que encontró en Internet sobre los niños con trastorno del espectro autista y que predecían que su hijo nunca sería independiente, ni se casaría, ni saldría de casa. Teme por la falta de independencia de Bentinho, ya que sabe que no vivirá para siempre y tiene la expectativa de que sus otros dos hijos formen una familia y, probablemente, no sean siempre responsables del cuidado de su hermano (estas proyecciones no están garantizadas en la realidad, pero forman parte del imaginario parental). Así, se refería a él como su hijo eterno: a los demás les crecerían alas y se irían de casa, pero él se quedaría, sin poder adquirir nunca autonomía en el camino de la vida.

Estas nociones se ven reforzadas, en gran medida, por la forma en que se considera la discapacidad como algo inherente al cuerpo o a la propia persona, y no como un aspecto relacional con el mundo. Cuando no se piensa en términos de normalidad, sino de normatividad -en el sentido que Canguilhem da al término-, el entorno, no solo biológico sino también en sus dimensiones ambiental y social, se vuelve esencial en la comprensión de la condición de la persona. Así, la propia noción de autonomía puede ser resignificada, cuando pensamos no en las limitaciones biológicas para realizar ciertas funciones o llevar a cabo ciertas actividades, sino en la diversidad de los cuerpos como parte de otra ética del cuidado ${ }^{(14)}$.

A pesar de ser profesionales de la salud con una historia de intensa defensa del SUS y de otras formas de producir cuidados, estas mismas dudas sobre la potencia de una persona discapacitada para caminar la vida también inundó el imaginario de la pareja Maria y José. Este último, a pesar de no manifestar un sentimiento de culpabilidad por el autismo de su hijo João, al asumir responsablemente la paternidad junto a Maria, también asume el reconocimiento ante sí mismo de que es padre de una persona con discapacidad y la necesidad de revisar las expectativas sobre lo que debe esperar de su hijo. La experiencia de responsabilidad de José es bastante singular en la realidad brasileña y paraibana, donde los niños suelen quedar huérfanos de padre, sobre todo tras un diagnóstico de discapacidad. Al asumir junto con su esposa la responsabilidad compartida del cuidado de João, José produce no solo una relación diferenciada como pareja, sino que también parece establecer otras formas de masculinidad en la percepción de otros padres y profesionales de la salud ${ }^{(15,16)}$.

Sin embargo, estas preocupaciones no parecen ser fundamentales para Rita. Su hijo con un diagnóstico de trastorno del espectro autista y un posible diagnóstico de altas capacidades/superdotado suele tener que adaptarse a la dinámica familiar. En ningún momento Rita manifiesta su preocupación por una posible incapacidad de su hijo para andar por la vida, ni actualmente ni en el futuro. Así, hay que tener en cuenta que la singularidad del diagnóstico de discapacidad (más o menos severa o socialmente estereotipada, producida por el capacitismo), así como la dinámica de cada familia y su experiencia con la discapacidad, producen diferentes formas de relacionarse con la discapacidad y con las expectativas sobre el poder de las personas con discapacidad para transitar la vida con autonomía y felicidad.

En este sentido, el capacitismo, como producción simbólica que parte de un imaginario y de un estándar corporal normatizado, con el apoyo intencional o no de toda la cultura, 
genera una narrativa sistematizada de opresión sobre los cuerpos de las personas con discapacidad, así como el racismo oprime los cuerpos de los afrodescendientes y el machismo explota los cuerpos de las mujeres ${ }^{(17)}$.

\section{El lugar de la discapacidad en la dinámica familiar}

La mayor o menor expectativa de los padres sobre la potencia de producción de vida de un niño o una niña con discapacidad no elimina el hecho de que la atención que demanda, especialmente en el proceso de diagnóstico y a lo largo de la primera infancia (donde se espera un mayor impacto de las estrategias rehabilitadoras y habilitadoras) desplaza a los cuidadores hacia otras y diferentes formas de cuidado.

En cualquier caso, la llegada de un miembro a la familia, con o sin discapacidad, produce la reinvención de la familia. La discapacidad, especialmente para las familias con poca o ninguna experiencia en este contexto, puede producir grandes interferencias. En el caso de las mujeres en general, hay estudios que muestran una mejor autoestima personal y colectiva cuando se convierten en madres, en comparación con las mujeres que no lo son, posiblemente por reafirmar los estereotipos tradicionales que consideran la maternidad como una dimensión esencial y necesaria para la plena condición femenina ${ }^{(18)}$. Sin embargo, es fundamental reconocer que esta percepción del papel de las mujeres en la maternidad y las propias formas de incorporar el cuidado materno a los procesos de subjetivación tienen una historia que, en gran medida, está conectada con las estrategias de medicalización de los cuerpos, buscando producir como efecto ciertos tipos de familias/individuos y no otros ${ }^{(19)}$.

Para Glória, Bentinho requiere más de su atención. Su nacimiento interfirió en su desempeño profesional en el sector de telecomunicaciones de la empresa familiar ya que, debido a las exigencias de cuidado de Bentinho, empezó a faltar mucho. A veces tenía que llevarlo al trabajo, lo que incomodaba a su marido, que se quejaba del ruido y del comportamiento "testarudo" del niño.

En una organización familiar marcada por el patriarcado, la madre es la que renuncia a su trabajo, y a menudo a su crecimiento profesional, para cuidar de su prole. Las mujeres que se reincorporan al trabajo tras la licencia de maternidad señalan no solo un mayor cansancio físico, debido a la realización simultánea de varias actividades que se acumulan con las del hogar, sino también por la exacerbación de la preocupación por sus hijos y/o hijas, entre otros elementos, por lo que resulta difícil equilibrar estas exigencias con los beneficios cognitivos, sociales y emocionales de sentirse productiva e incluida en el mundo laboral(20). Las madres de las personas con discapacidad necesitan añadir a tales retos la dificultad de lidiar con las diferentes sensaciones -que son más intensas en el período inicial que reciben el diagnóstico- para reorganizar sus perspectivas, en las que se mencionan sentimientos de perplejidad, vergüenza, humillación, además de la pérdida de relaciones y referencias que ayudaban a dar sentido a su mundo cultural y social(21).

En la dinámica familiar de Glória, su hija mujer también perdió gran parte de su juventud para ayudar a cuidar a su hermano Bentinho mientras su madre trabajaba. Cuando la situación se volvió insostenible para madre e hija, Glória dejó su trabajo y empezó a desempeñar un papel destacado en la organización de la ONG que había fundado y que se convirtió en el centro de su vida dentro y fuera del ámbito familiar/doméstico. Si el cuidado de Bentinho, al principio ocupaba la vida de las mujeres de la casa, poco a poco su diagnóstico y la producción de redes relacionales centradas en el trastorno del espectro autista ganaron protagonismo en la dinámica familiar en su conjunto. Este desplazamiento no es inusual e incluso reafirma la narrativa de varias mujeres que resignifican su relación consigo mismas y con la familia a partir de la experiencia de la maternidad de personas con discapacidad, asumiendo el rol de protagonistas en la construcción de redes de cuidado y protección, así como en la lucha por garantizar derechos para sus hijos e hijas ${ }^{(22,23)}$. 
La asociación destinada a apoyar a las madres de niños con trastorno del espectro autista, empieza a demandar más atención familiar hasta el punto de eclipsar el protagonismo de Bentinho. Glória habla muy poco o casi nada de él en nuestras conversaciones. En sus relatos predomina la discusión sobre la asociación y/o su activismo social. La conversación sobre Bentinho se centra en el relato del embarazo, parto y puerperio de Glória hasta el descubrimiento del diagnóstico de trastorno del espectro autista, cuando su hijo tenía dos años. Cada vez que él insiste en aparecer en nuestras videoconferencias semanales, Glória lo aparta bruscamente alegando que interrumpe el flujo de sus ideas, y a menudo exige que su hija se ocupe de él lejos de las cámaras.

A partir de la experiencia con Bentinho y la asociación (es decir, con el trastorno del espectro autista y las personas que viven $y$ conviven con él), todos los miembros de la familia comenzaron a estudiar psicología en la misma universidad privada. Para Glória y su marido, que se casaron en la adolescencia y vieron postergado su sueño de acceder a la educación superior, esta fue su primera posibilidad de estudiar. Sin embargo, su hija estaba cursando el $5^{\circ}$ semestre de Ingeniería Alimentaria en una universidad federal del mismo estado, y su hijo, el $7^{\circ}$ semestre de Ingeniería Civil en una universidad federal de un estado cercano, y ambos se trasladaron para estudiar psicología. Además, Glória y sus dos hijos se han formado en el manejo de la terapia Ilamada Applied Behavior Analysis (ABA) y la desarrollan con los niños y las niñas de la asociación.

Lo que hemos visualizado de forma más emblemática en la organización familiar de Glória también se puede visualizar, en mayor o menor medida, en otras dinámicas familiares que hemos mapeado. Maria y José relataron que la llegada de João y sus nuevas exigencias de cuidado hacían imposible que ambos continuaran con un proyecto profesional en Brasilia. Tuvieron que volver a João Pessoa para asegurar una red de cuidados para su hijo (ya sea de oferta de servicios o de apoyo familiar) y para ello tuvieron que iniciar nuevas trayectorias profesionales. Maria, que ya había sido profesora de Salud Colectiva en un centro de enseñanza superior, vuelve a dar clases y a trabajar en la atención clínica individual especializada en la atención a niños con trastorno del espectro autista, y se convierte cada vez más en una referencia en la atención a personas con esta condición. También creó proyectos de extensión e incluyó, en los cursos de licenciatura de la institución privada en la que enseña, acciones en torno a este tema, que la llevó a ser aún más reconocida por sus colegas profesores. En cierto modo, este diagnóstico también reorientó sus estudios y su desempeño profesional, así como su cuidado diario de João en varios servicios e instituciones.

En el contrapunto de esta forma de organizar la familia, encontramos a Rita que, tras unos meses guiada por el trastorno del espectro autista, es decir, estudiando, investigando y relacionándose casi exclusivamente a partir de este dispositivo diagnóstico, decide retomar sus proyectos personales de vida, que van más allá de ser la madre de Joaquim, un niño con trastorno del espectro autista. La familia se ve afectada por la presencia de Joaquim, pero no se siente subordinada a sus necesidades de cuidado. La familia busca una dieta con menor consumo de proteínas animales y lactosa (recomendada para niños con trastorno del espectro autista), pero no asume una dieta totalmente vegana, por ejemplo. En la misma dirección, Joaquim, que no soportaba el contacto con la arena de la playa, fue exponiéndose poco a poco a este entorno, hasta el punto de que hoy ya no le molesta, porque la playa es uno de los lugares de ocio favoritos de su familia. Tras completar su maestría, Rita desea realizar un doctorado. Justifica sus proyectos personales alegando que siendo mejor persona podrá ser mejor madre para sus hijos, y así negocia sus sueños con las exigencias sociales e imaginarias de la madre ideal ${ }^{(23)}$.

\section{La producción de núcleos específicos de dependencia relacional}

La producción de autonomía en la forma de transitar la vida está directamente relacionada 
con la producción de redes de cuidado, es decir, redes relacionales de dependencia. Cuanto más grandes, potentes y efectivas son las redes de dependencias relacionales que produce una persona, mayor es la movilidad de vida que también es capaz de producir. Lo que observamos en los procesos institucionalizados de cuidado de la salud es que el cuidado se organiza centrado en las ofertas de las instituciones y servicios de salud, y debilita otras redes relacionales de las personas ${ }^{(24)}$.

Hemos aprendido en el campo de la salud mental que la institucionalización del cuidado ha situado la locura en el centro de la producción de la existencia de las personas. Así, una persona deja de tener visibilizadas todas sus multitudes existenciales (esposa, madre, hermana, trabajadora, etc.) para ser una persona con esquizofrenia, por ejemplo. Todas sus formas de caminar por la vida estarán guiadas o reducidas por este diagnóstico clínico que aprisiona su producción existencial(25).

Al analizar el cuidado de las personas con discapacidad hemos visto algo similar, en un proceso de centralización del diagnóstico de la discapacidad en la producción subjetiva-existencial de las personas e incluso en hacer efectivos sus derechos. El diagnóstico se convierte en un dispositivo imperativo de procesos y relaciones, organizando de forma estructurante la forma de vivir en comunidad de la persona ${ }^{(14)}$. Esta lógica acaba obstruyendo la capacidad de identificar qué otras necesidades son esenciales, no solo en términos de salud, sino más allá de la salud, como la garantía de los derechos, el reconocimiento de la ciudadanía, el acceso a la educación, las oportunidades de ocio, la accesibilidad en el transporte, la producción artística y cultural, entre varias otras dimensiones de la vida de las personas ${ }^{(7)}$.

$\mathrm{Al}$ igual que en la salud mental, en el ámbito de la discapacidad, el diagnóstico clínico opera no solo sobre la vida de las personas con discapacidad/locos, sino también sobre la vida de las personas que conviven con ellas, es decir, que comparten parte de sus territorios existenciales. La familia, como red más cercana de la persona con discapacidad/loco, queda entonces marcada por el diagnóstico en su dinámica de funcionamiento, ya sea negándolo o convirtiéndolo en un referente de la vida cotidiana familiar, como vimos en el analizador anterior, en el que el diagnóstico de trastorno del espectro autista estructura el modo de vida de las familias.

Sin embargo, es interesante observar que este registro del diagnóstico afecta la producción de sus redes relacionales, que pueden ser reforzadas o empobrecidas. En la experiencia de Glória, el diagnóstico de trastorno del espectro autista de Bentinho asumió una importante centralidad en la dinámica familiar, hasta el punto de orientar la mayor parte de su producción de redes relacionales vivas. La creación de la asociación, así como la carrera de Psicología, se centraron en la necesidad de apoyar a las madres de autistas del CER IV con las que Glória convivía y se identificaba en el dolor o el peso de enfrentarse a tantas barreras de acceso en la búsqueda de cuidados. La asociación nació en los asientos de la recepción del CODEI/SERI, es decir, mientras esperaba, junto con otras madres, que sus hijos fueran atendidos, y que compartían el sufrimiento y las dificultades en el proceso de cuidado, en un contexto social excluyente, marcado por diversas barreras económicas, de comunicación, pedagógicas, actitudinales y tecnológicas.

Tanto en la recepción del CODEI/SERI, como en los pasillos del CER IV, trabajadores, gestores y usuarios reconocen los escenarios de encuentros de personas que viven y conviven con la discapacidad, especialmente mujeres, en los que se construyen (y deconstruyen) relaciones de afecto, sororidad y apoyo, ya que estas mujeres se conocen, se encuentran y producen redes relacionales por y para la discapacidad que marcan los cuerpos de sus hijos. Lo que llama la atención es que, por un lado, si bien estos encuentros con otras personas dentro de los muros del CER IV han potenciado la producción de redes relacionales, por otro lado, han hecho visible que estas han sido las principales (y casi únicas) redes de sociabilidad de estas personas. En otras palabras, la producción relacional de las personas con discapacidad y de sus 
familias se centra en las conexiones con otras personas con la misma discapacidad, intensificando el funcionamiento de este signo en la experiencia de vida de las personas. En parte, esto puede deberse a la necesidad de afrontar los impactos de la identificación de una persona con discapacidad en la familia que, entre otros efectos, incluye un amplio conjunto potencial de sentimientos como el miedo, la angustia, la inseguridad, la frustración, la ira y el amor ${ }^{(26)}$. Y, en medio de todo esto, compartir la experiencia de tener un hijo o una hija con discapacidad promueve el reconocimiento, la acogida y el apoyo, la mayoría de las veces, de otras familias en esta situación que, al compartir sus relatos, dan un nuevo sentido a sus existencias, reavivan las esperanzas y reposicionan expectativas ${ }^{(21)}$.

Dando forma a este analizador, Glória crea su asociación para estar con madres y familiares de personas con trastorno del espectro autista, es decir, con personas con las que comparte una experiencia de vida. Ella nos cuenta que las otras personas que no cohabitan este territorio existencial suyo (personas con discapacidad) ya no la "aguantaban" con sus intereses y conversaciones, que siempre giraban en torno a sus caminos en la red personas con discapacidad en busca de asegurar el cuidado de Bentinho.

Maria y José también comenzaron a producir nuevas redes relacionales tras el diagnóstico de trastorno del espectro autista de João. Desde el momento en que empezaron a tener dificultades para mediar las relaciones cotidianas con otros padres en los espacios públicos y en las escuelas regulares, comenzaron a conectarse más con redes de madres de niñas y niños autistas, lo que permitió la circulación de experiencias y afectos, y la experimentación de otras formas de cuidado no medicalizantes. A través de medios presenciales y virtuales, estos grupos articulan amplias redes de apoyo social del ámbito local, regional e incluso interestatal. Además, Maria, y en menor medida José, se involucraron intensamente en las actividades y en la gestión de una organización de la sociedad civil (distinta de la asociación de Glória) que busca producir otras formas de atención a las personas con trastorno del espectro autista, centrándose en los fenómenos paralelos que desencadenan o intensifican el autismo (como los procesos inflamatorios basados en una alimentación inadecuada), entre otros.

En la misma dirección, Rita evalúa que la mayor parte de sus interacciones sociales se centra en las relaciones familiares y profesionales y en las que produjo a partir del encuentro con otras madres de niños y niñas con trastorno del espectro autista. Incluso sus redes más antiguas, producidas antes de la llegada de Joaquim, están hoy atravesadas por personas que también tuvieron hijos diagnosticados con trastorno del espectro autista. Parece que hay un cierto inconveniente a la hora de estar en espacios o relaciones con personas que no comparten este territorio existencial (personas con discapacidad), porque es más angustioso relacionarse con ellos, debido al capacitismo que (re)guía las relaciones sociales no mediadas por este signo.

En la experiencia de Margarida, que no produjo conexiones más amplias con otras personas que viven o conviven con la discapacidad visual, su red relacional se volvió extremadamente restringida, limitada a su familia nuclear (padres, marido e hijo), a los rehabilitadores y a otras personas con discapacidad usuarias del CODAVI del mencionado CER IV, considerando que las amistades que tenía anteriormente desaparecieron después de que Margarida empezó a presentar baja visión.

\section{Las imbricaciones público vs. privado en las ofertas de cuidados de las personas con discapacidad}

Al igual que en otros ámbitos de la asistencia especializada en salud, la red de atención a las personas con discapacidad se constituye, en gran medida, mediante un entramado de lo público y lo privado. Por un lado, porque una parte considerable de las acciones y servicios especializados en el país, históricamente, son realizados por servicios privados contratados por el poder público para atender a los usuarios del SUS ${ }^{(27)}$. Por otro lado, por el protagonismo de las personas que, en la construcción 
de sus redes de cuidado, cuando logran acceder y por diferentes vías, conectan las ofertas de servicios públicos y privados constituyendo mapas únicos, según los planes de cuidado que ellas mismas construyen sobre la base de sus proyectos de vida ${ }^{(28)}$.

Glória evalúa, por ejemplo, que el tratamiento ofrecido por el CER IV era insuficiente para la evolución de Bentinho y que necesitaba más actividades o terapias. Ella entiende la limitación del CER IV y sus estrategias para producir barreras en la continuidad del cuidado para dar acceso a otras personas que no cuentan con ningún tipo de cuidado. Bentinho contó con el seguimiento de varios profesionales, pero con el aumento de la demanda en el CODEI/SERI, su tiempo de atención se redujo de 45 a 30 minutos y, a medida que fue creciendo, fue desvinculado de algunas terapias. Además, según ella, el CER IV no ofrece la modalidad terapéutica ABA por falta de tiempo de los profesionales, y por la necesidad de atender la demanda reprimida del servicio. Glória también se enorgullece de que su asociación ofrezca diversas actividades para las familias, financiándose con donaciones, articulaciones con la intendencia municipal de João Pessoa y la sociedad civil, sin necesidad de cobrar valores monetarios elevados, como hacen otras asociaciones (que también cuentan con profesionales contratados por el municipio).

Al mismo tiempo, Maria y José dejaron Brasilia porque no podían acceder a servicios públicos de calidad y la red privada era demasiado cara, incluso para una pareja con estudios universitarios que trabajaba para el gobierno federal. En João Pessoa, ante las dificultades para acceder a los servicios sanitarios públicos, que siempre han sido su principal bandera de lucha política, acceden a gran parte de los servicios privados a través de los seguros de salud, siendo difícil mantener financieramente todas las acciones asistenciales necesarias. También insatisfechos con las modalidades de asistencia desarrolladas en João Pessoa, encontraron en Recife un servicio privado que realiza la atención que consideraron más adecuada, pero que genera dificultad de acceso debido a la necesidad de asumir los altos costos implicados. Además, Maria y José se refieren al modo depredador de la atención médica para las personas con trastorno del espectro autista en el sector privado, también atravesado por una fuerte judicialización. Por un lado, denuncian la existencia de servicios que facilitan la emisión de sentencias, a través de profesionales que cobran cantidades desorbitadas por ello. Por otro, que los propios seguros de salud, debido a los elevados costos en reiteradas derrotas judiciales, en las que se ven obligados a ofrecer las más amplias acciones de asistencia, están construyendo ofertas de atención precarias, para desalentar a las familias a recurrir a la justicia en busca de más ofertas.

Rita, por su parte, a pesar de señalar la calidad del CER IV en el diagnóstico y en las terapias de rehabilitación, reconoce que la red pública está sobrecargada por la alta demanda. Así, va al CER IV para obtener los informes necesarios para acceder a los derechos sociales de su hijo, pero prefiere realizar las terapias desde la red privada asociada al plan de salud. Reconoce que hay varias acciones y profesionales especializados en trastorno del espectro autista que no puede costear. Así, construye el proyecto terapéutico de Joaquim a partir de sus posibilidades económicas.

En las narraciones de las tres madresciudadanas-guías de este estudio, es posible constatar que existe, en la red privada, la producción de una multitud de ofertas terapéuticas, que producen un importante mercado de orientación capitalista. Maria y José señalan incluso que unas pocas personas tienen acceso a servicios súper individualizados, con equipos enteros altamente especializados en técnicas avanzadas de atención a niños y niñas con trastorno del espectro autista que vienen de São Paulo periódicamente a João Pessoa, con pasajes aéreos y hospedaje pagados por familias de alto poder adquisitivo. Estos profesionales se ponen a disposición durante toda una semana para atender a grupos muy restringidos de niños. Se trata de acciones terapéuticas, obviamente, de alcance limitado a una pequeña porción de la población, lo que denuncia la existencia de una flagrante diferencia de acceso debido a 
la enorme concentración de ingresos y a la desigualdad de clase en nuestro país.

En cuanto a Margarida, como ya se ha mencionado, la red privada fue su primera opción para la cirugía oftalmológica, aunque le costó un dinero que no tenía. Con el fracaso de la primera cirugía (en la red privada) su acceso a la red pública se vio impregnada de incredulidad y desvalorización de las ofertas de atención del SUS. Incluso con la experiencia exitosa de la nueva cirugía realizada por la red pública, Margarida todavía mantiene reticencias sobre el SUS, a pesar de mantener un vínculo con el CER IV y el desarrollo de algunas terapias.

\section{SOBRE EL CAMINO RECORRIDO HASTA AHORA}

Este conjunto de reflexiones, desarrollado a partir de las situaciones identificadas en la relación entre nosotros, investigadores del GPECS, las usuarias-ciudadanas-guías y sus familias, nos permitió analizar algunos elementos sobre la producción de la red de cuidados de las personas con discapacidad en João Pessoa que, en parte, dialoga con otras localidades.
Los analizadores aquí presentados, más que contemplar todos los análisis posibles, nos invitan a profundizar en las trayectorias singulares de las personas con las que hemos desarrollado esta investigación, para que las reflexiones realizadas con ellas sobre sus experiencias y conocimientos puedan ayudar aún más a pensar el cuidado producido. Además, destacamos que aquí optamos por profundizar en el análisis, tomando casi exclusivamente el punto de vista de las usuarias-ciudadanas-guías; por lo tanto, es importante cruzar otros análisis que permitan un caleidoscopio más amplio con las visiones desde los puntos de vista de otros productores de redes de cuidados de personas con discapacidad. Las cuestiones aquí planteadas se vuelven aún más complejas cuando las confrontamos con lo que estas reflexiones les permiten ver y decir a los trabajadores y gestores de la salud, desde sus diferentes ámbitos, y esa complejidad de perspectivas simétricas puede potenciar mucho de lo que acabamos de señalar en este artículo. Tales aspectos, sin embargo, no figuraban entre los objetivos que nos propusimos en la presente elaboración, quedando para ser formulados en estudios posteriores.

\section{AGRADECIMIENTOS}

Este artículo forma parte del proyecto de investigación, en ejecución, titulado Análise da Implantação da Rede de Cuidados à Saúde das Pessoas com Deficiência (RCPCD) - os usuários, trabalhadores e gestores como guias, aprobado en la convocatoria pública de proyectos CNPq/MS/SCTIE/DECIT/SAS/DAPES/CGSPCD No. 35/2018 - Avaliação da Implementação da Rede de Cuidados à Saúde das Pessoas com Deficiência no Sistema único de Saúde (SUS), con financiamiento autorizado por el Conselho Nacional de Desenvolvimento Científico e Técnologico, proceso No. 442816/2018-9, iniciado en el año 2018 y aún vigente, bajo la coordinación del profesor Dr. Emerson Elias Merhy, de la Universidade Federal do Rio de Janeiro (UFRJ).

\section{REFERENCIAS BIBLIOGRÁFICOS}

1. Kuschnir R, Chorny $A H$. Redes de atenção à saúde: contextualizando o debate. Ciência \& Saúde Coletiva. 2010;15(5):2307-2316. doi: 10.1590/S141381232010000500006 .
2. Merhy EE. As vistas dos pontos de vista. Tensão dos programas de Saúde da Família que pedem medidas. Revista Brasileira de Saúde Família. 2013;15(35/36):1-7.

3. Viveiros de Castro E. Metafísicas Canibais: elementos para uma antropologia pós-estrutural. São Paulo: Cosac Naify, n-1 edições; 2015.

4. Moebus RLN, Merhy EE, Silva E. O Usuário-cidadão como guia: Como pode a onda elevar-se acima da montanha. En: Merhy EE, Baduy RS, Seixas CT, Almeida DES, Slomp Júnior H (orgs). Avaliação compartilhada do cuidado em saúde: surpreendendo o instituído nas redes. Rio de Janeiro: Hexis; 2016. p. 43-53.

5. Gomes MPC, Merhy EE. Pesquisadores in-mundos: um estudo da produção de acesso e barreira em saúde mental. Porto Alegre: Editora Rede Unida; 2014.

6. Cecilio LCO, Carapinheiro G, Andreazza R, Souza ALM, Andrade MGG, Santiago SM, et al. O agir leigo e o cuidado em saúde: a produção de mapas de cuidado. Cadernos de Saúde Pública. 2014;30(7):1502-1514. doi: 10.1590/0102-311X00055913. 
7. Othero MB, Ayres JRCM. Necessidades de saúde da pessoa com deficiência: a perspectiva dos sujeitos por meio de histórias de vida. Interface - Comunicação, Saúde, Educação. 2012;16(40):219-234. doi: 10.1590/ S1414-32832012005000010.

8. Vilarinho-Rezende D, Fleith DS, Alencar EMLS. Desafios no diagnóstico de dupla excepcionalidade: um estudo de caso. Revista de Psicología. 2016;34(1):61-84 doi: 10.18800/psico.201601.002.

9. Tiburi M. Feminismo em comum: para todas, todes e todos. Rio de Janeiro: Rosa dos Tempos; 2018.

10. Welter I, Cetolin SF, Trzcinski C, Cetolin SK. Gênero, maternidade e deficiência: representação da diversidade. Textos Contextos (Porto Alegre). 2008;7(1):98-119

11. Gonçalves AP, Silva B, Menezes M, Tonial L. Transtornos do espectro do autismo e psicanálise: revisitando a literatura. Tempo Psicanalitico. 2017:49(2):152-181.

12. Iriart C, Merhy EE. Disputas inter-capitalistas, biomedicalización y modelo médico hegemónico. Interface - Comunicação, Saúde, Educação. 2017;21(63):10051016. doi: 10.1590/1807-57622016.0808.

13. Almeida MB. O filho eterno: uma leitura desejante. Revista Estudos Feministas. 2009;17(1):265-276. doi: 10.1590/S0104-026X2009000100017.

14. Gaudenzi P, Ortega, F. Problematizando o conceito de deficiência a partir das noções de autonomia e normalidade. Ciência \& Saúde Coletiva. 2016;21(10):30613070. doi: 10.1590/1413-812320152110.16642016.

15. Machin R, Couto MT, Silva GSN, Schraiber LB, Gomes R, Santos FW, et al. Concepções de gênero, masculinidade e cuidados em saúde: estudo com profissionais de saúde da atenção primária. Ciência \& Saúde Coletiva. 2011;16(11):4503-4512. doi: 10.1590/S141381232011001200023.

16. Schraiber LB, Figueiredo WS, Gomes R, Couto MT, Pinheiro TF, Machin R, et al. Necessidades de saúde e masculinidades: atenção primária no cuidado aos homens. Cadernos de Saúde Pública. 2010;26(5):961970. doi: 10.1590/S0102-311X2010000500018.

17. Campbell FK. Inciting legal fictions: disability's date with ontology and the ableist body of the law. Griffith Law Review. 2001;10(1):42-62.
18. Souza DBL, Ferreira MC. Auto-estima pessoal e coletiva em mães e não-mães. Psicologia em Estudo. 2005;10(1):19-25.

19. Moura SMSR, Araújo MF. A maternidade na história e a história dos cuidados maternos. Psicologia: Ciência e Profissão. 2004;24(1):44-55. doi: 10.1590/S141498932004000100006.

20. Garcia CF, Viecili J. Implicações do retorno ao trabalho após licença-maternidade na rotina e no trabalho da mulher. Fractal: Revista de Psicologia. 2018;30(2):271 280. doi: 10.22409/1984-0292/v30i2/5541.

21. Bastos OM, Deslandes SF. A experiência de ter um filho com deficiência mental: narrativas de mães. Cadernos de Saúde Pública. 2008;24(9):2141-2150. doi: $10.1590 /$ S0102-311X2008000900020.

22. Lopes BA. Autismo, narrativas maternas e ativismo dos anos 1970 a 2008. Revista Brasileira de Educação Especial. 2020;26(3):511-526. doi: 10.1590/1980$54702020 v 26 \mathrm{e} 0169$.

23. Badinter E. Um amor conquistado: o mito do amor materno. Rio de Janeiro: Nova Fronteira; 1985.

24. Merhy EE. Cuidado com o cuidado em saúde: saber explorar seus paradoxos para um agir manicomial. En: Merhy EE, Amaral H, orgs. Reforma psiquiátrica no cotidiano II. São Paulo: Hucitec; 2007.

25. Lancetti A. Clínica Peripatética. São Paulo: Hucitec 2005.

26. Dantas MSA, Collet N, Moura FM, Torquato IMB. Impacto do diagnóstico de paralisia cerebral para a família. Texto \& Contexto - Enfermagem. 2010;19(2):229-237. doi: 10.1590/S0104-07072010000200003.

27. Solla J, Chioro A. Atenção ambulatorial especializada. En: Giovanella L, Escorel S, Lobato LVC, Noronha JC, Carvalho Al, orgs. Políticas e sistemas de saúde no Brasil. Rio de Janeiro: Fiocruz; 2008. p. 627-663.

28. Meneses CS, Cecilio LCO, Andreazza R, Carapinheiro G, Andrade MGG, Santiago SM, et al. O agir leigo e a produção de mapas de cuidado mistos público-privados. Ciência \& Saúde Coletiva. 2017;22(6):2013-2024. doi: 10.1590/1413-81232017226.14822016.

\section{FORMA DE CITAR}

Sampaio J, Barbosa DS, Gomes LB, Peixoto DA, Menezes TA, Zózimo HHL, Lyra MFB. Cartografiar el tejido de redes de atención para personas con discapacidad, desde la perspectiva de usuarias-ciudadanas-guías. Salud Colectiva. 2021;17:e3334. doi: 10.18294/sc.2021.3334.

Recibido: 6 dic 2020 | Versión final: 8 mar 2021 | Aprobado: 9 mar 2021 | Publicado en línea: 7 jun 2021

Esta obra está bajo una licencia de Creative Commons Reconocimiento-NoComercial 4.0 Internacional. Reconocimiento - Permite copiar, distribuir y comunicar públicamente la obra. A cambio, se debe reconocer y citar al autor original. No Comercial - Esta obra no puede ser utilizada con finalidades comerciales, a menos que se obtenga el permiso. 